\title{
Actividad sérica de la acetilhidrolasa del factor activador de plaquetas en pacientes afrodescendientes y mestizos con dengue, Colombia
}

\author{
Berta Nelly Restrepo', Margarita Arboleda1, Ruth Ramirez, Gonzalo Álvarez', 2 \\ 1 Instituto Colombiano de Medicina Tropical-Universidad CES, Medellín, Colombia \\ 2 Grupo Malaria, Universidad de Antioquia, Medellín, Colombia
}

Introducción. El dengue grave se caracteriza por el aumento de la permeabilidad vascular inducida por citocinas y mediadores químicos; uno de estos es el factor activador de plaquetas. La acetilhidrolasa del factor activador de plaquetas es la enzima responsable de su degradación, y su deficiencia o aumento se ha relacionado con varias enfermedades. Sin embargo, su papel en la infección por dengue es poco conocido, como tampoco se sabe si existe diferente actividad según el grupo étnico.

Objetivo. Comparar la actividad en suero de la acetilhidrolasa del factor activador de plaquetas en dos grupos étnicos con infección por el virus del dengue.

Materiales y métodos. Se llevó a cabo un estudio descriptivo, longitudinal, prospectivo, en dos departamentos de Colombia, Antioquia y Chocó. A 43 pacientes mestizos y a 33 pacientes afrodescendientes con diagnóstico de dengue, se les tomó una muestra de suero por cinco días consecutivos en la fase aguda y una muestra en la de convalecencia.

Resultados. Se observó mayor frecuencia de casos de dengue hemorrágico en los pacientes mestizos que en los afrodescendientes $(23,3 \%$ Vs. $12,1 \%, p=0,248)$. La actividad sérica de la acetilhidrolasa del factor activador de plaquetas (mediana, percentil 25 y percentil 75) fue más elevada en afrodescendientes que en mestizos $[0,89(0,72-1,10)$ Vs. 0,76 $(0-1,03), p=0,000]$. Este comportamiento se conserva en el dengue clásico $[0,89(0,73-1,10)$ Vs. $0,73(0-1,05), p=0,000]$ y en el hemorrágico $[0,88(0,69-1,12) V s$. $0,83(0,71-1,08), p=0,893]$.

Conclusiones. Se encontró mayor producción de acetilhidrolasa del factor activador de plaquetas en los pacientes afrodescendientes. Sin embargo, es necesario hacer estudios de polimorfismos de esta enzima que permitan obtener resultados concluyentes.

Palabras clave: 1-alquil-2-acetil-glicerofosfocolina esterasa, dengue, grupos étnicos, Colombia.

Serum platelet-activating factor acetylhydrolase activity in dengue patients of African or mestizo descendency

Introduction: Severe dengue infection is characterized by enhanced vascular permeability produced by cytokines and biochemical mediators. One of these mediators is the platelet-activating factor. Degradation of platelet-activating factor is accomplished by its specific acetylhydrolase. Down or up regulation of this enzyme has been linked to several diseases. However, the role of this enzyme in dengue infection is unknown, a well as whether differential activity occurs by ethnic group.

Objective: The activity of the platelet-activating factor-acetylhydrolase was compared in patients infected with dengue virus in two ethnic groups.

Material and methods: A descriptive, prospective and longitudinal study was made in two states of Colombia (Antioquia and Chocó). Serum samples were taken from 43 mestizo patients and 33 patients of African descent, each taken during five consecutive days of the acute dengue phase. A second serum sample was taken during the convalescent phase.

Results: The mestizo patients showed higher frequency of dengue hemorrhagic fever than the patients of African descent $(23.3 \%$ vs. $12.1 \%, p=0.25)$. The serum activity of the platelet-activating factor acetylhydrolase was higher in African descendents than in mestizos (0.89, Cl: 0.72-1.10 vs. 0.76 $\mathrm{Cl}: 0-1.03, p<0.001)$. This relationship is maintained for occurence of dengue fever $(0.89, \mathrm{Cl}: 0.73-1.10$ vs. $0.73, \mathrm{Cl}: 0-1.05, p<0.001)$, but not significant for dengue hemorrhagic fever $(0.88, \mathrm{Cl}: 0.69-1.12$ vs. 0.83, Cl: $0.71-1.08, p=0.89$ ).

\section{Contribución de los autores:}

Berta Nelly Restrepo: idea de la investigación, escritura del proyecto, análisis de la información y escritura del manuscrito. Margarita Arboleda: captación de pacientes en la región de Urabá y revisión del artículo.

Ruth Ramírez: elaboración de la bases de datos y procesamiento de las muestras de laboratorio.

Gonzalo Álvarez: análisis de la información y escritura del manuscrito. 
Conclusion. An increased production of platelet-activating factor-acetylhydrolase occurs in the serum of dengue-infected African descendants. However, a study of the frequencies of polymorphisms for this enzyme will permit more conclusive support for these observations.

Key words: 1-alkyl-2-acetylglycerophosphocholine esterase, dengue, ethnic groups, Colombia

El dengue es un importante problema de salud pública en 112 países tropicales y subtropicales. Cada año ocurren 50 millones de infecciones por dengue en el mundo (1). Durante el quinquenio 2005-2009, se registraron, en promedio por año, 44.268 casos de dengue en Colombia, 5.520 de los cuales eran dengue hemorrágico. Sin embargo, durante el 2010, esta frecuencia aumentó en $230 \%$, reportándose para este año 146.354 casos de dengue (2), con circulación simultánea de los cuatro serotipos, con predominio de DENV-2 (44\%) y DENV-1 (41\%) (3).

La infección por el virus del dengue es asintomática en la mayoría de los casos, o puede cursar de forma leve y benigna -dengue clásico- o evolucionar a formas graves-dengue hemorrágicocaracterizadas por presentar extravasación plasmática, trombocitopenia y hemorragias (4). Estas manifestaciones son el resultado de las alteraciones del endotelio vascular (5).

La variedad del espectro clínico del dengue está determinada por interacciones entre los factores virales, la presencia de infección secundaria, la respuesta inmunológica, y los factores genéticos y sociales, no completamente entendidos. Se ha descrito que las formas graves del dengue están relacionadas con la virulencia de la cepa $(6,7)$, los serotipos DENV-1 y DENV-2, $(8,9)$, una mayor carga viral (10) y la circulación simultánea de varios serotipos (11).

Halstead postuló la teoría del aumento de la infección dependiente de anticuerpos (12). Durante una infección secundaria, los anticuerpos preexistentes forman, con los antígenos virales de la nueva infección, un complejo antígenoanticuerpo que permite el ingreso del virus a la célula a través de la fracción cristalizable y la subsecuente replicación viral. En respuesta, las células T CD4+ y CD8+ producen citocinas (13)

$\overline{\text { Correspondencia: }}$

Berta Nelly Restrepo, Instituto Colombiano de Medicina Tropical-Universidad CES, Carrera 43A № 52S-99, Sabaneta, Colombia

Teléfono: (574) 305 3500, extensión 2293; fax: (574) 3014258 brestrepo@ces.edu.co

Recibido: 01/03/11; aceptado:13/09/11 implicadas en el aumento de la permeabilidad vascular determinante de las manifestaciones de extravasación (14-18).

Por otro lado, la respuesta a las infecciones está influida por los antecedentes genéticos del huésped. Esta influencia se ha sugerido con relación a la raza. En Haití (19) y en África (20) no se reporta dengue hemorrágico. En un estudio reciente en Haití, se encontró una elevada prevalencia de anticuerpos contra el virus del dengue $(65 \%)$ en niños de 7 a 36 meses; sin embargo, la enfermedad grave no es un problema importante en la población autóctona y, como el autor lo afirma, posiblemente, los haitianos están, genéticamente o de alguna manera, protegidos de las formas graves (21). En Cuba, los descendientes africanos presentan formas menos graves de dengue (22-24). En Malasia, los chinos han registrado mayor incidencia de dengue hemorrágico que los malayos (25).

Como se mencionó anteriormente, se ha estudiado la relación de las citocinas con las formas graves del dengue, pero el papel de los mediadores lipídicos, como el factor activador de plaquetas, no se ha abordado en forma suficiente.

El factor activador de plaquetas (1-O-alquil-2acetil-sn-glicero-3-fosfocolina) es un fosfolípido producido por una variedad de células, como los neutrófilos, los eosinófilos, los macrófagos, los mastocitos y las células endoteliales, después de una estimulación química o inmunológica. Este mediador juega un papel importante en las reacciones alérgicas e inflamatorias (26), y actúa como una molécula de señalización intercelular y vascular en los procesos inflamatorios (27). Su función es estimular la agregación plaquetaria y leucocitaria, la contracción del músculo liso y la vasodilatación (28), así como el aumento de la permeabilidad vascular y, dependiendo de su regulación, puede conducir a diferentes estados de la enfermedad (27).

El factor activador de plaquetas (FAP), en su forma biológicamente inactiva es mediado por la enzima acetilhidrolasa del factor activador de plaquetas (AH-FAP) $(26,28,29)$. Esta enzima, también denominada lipoproteína-PLA2, (lipoprotein-associated 
phospho-lipase A2, Ip-PLA2), degrada los productos de la oxidación de fosfatidilcolina producidos por las lipoproteínas de baja densidad y del estrés oxidativo; por lo tanto, se considera una enzima potencialmente antiinflamatoria y, dependiendo de su concentración y de la disponibilidad de sustratos potenciales, puede poseer una doble función proinflamatoria y antiinflamatoria (30).

Existen tres tipos de AH-FAP, dos intracelulares (I y II) y una en plasma. Se considera que la de tipo II, así como la del plasma, tienen un papel "limpiador" de los fosfolípidos oxidados que se cree que participan en diversos procesos patológicos (31).

Se ha observado deficiencia de esta enzima en el plasma en casos de enterocolitis necrosante (32), sepsis $(33,34)$, asma (35), hiperlipidemia, enfermedad cardiovascular, artritis reumatoidea, enfermedad inflamatoria intestinal (28) y hepatitis $C$ $(36,37)$; y su aumento se ha asociado a hipertensión arterial (38), accidente cerebrovascular (39), deficiencia familiar de lipoproteínas de alta densidad (High Density Lipoprotein, HDL) y diabetes mellitus (40). Así mismo, la medición de la actividad de la AH-FAP en el plasma o suero, se considera una herramienta de diagnóstico clínico (26).

Con relación a la infección por el virus del dengue, Yang, et al., reportaron niveles más elevados del factor activador de plaquetas en los leucocitos mononucleares de pacientes con infección secundaria, que en los de aquellos con infección primaria (41). Además, observaron que el factor activador de plaquetas puede inducir el consumo de plaquetas y leucocitos que se encuentran en el dengue hemorrágico, y sugieren que este factor puede participar en su patogenia. En experimentos con ratones se ha demostrado que la activación sistémica del receptor del factor activador de plaquetas se asemeja a los cambios observados durante los casos graves de dengue, en comparación con los ratones sin el receptor (42). Seet, et al., señalaron que la actividad de la AH-FAP durante la fase febril de la infección por el virus del dengue, es elevada en comparación con la de la fase de convalecencia (43).

El presente estudio tuvo como objetivo comparar la actividad de la AH-FAP entre dos grupos étnicos (mestizos y afrodescendientes) con infección por el virus deldengue, procedentes deláreametropolitana de Medellín en el departamento de Antioquia y del municipio de Quibdó en el departamento de Chocó. De igual modo, se comparó la actividad de la $\mathrm{AH}$ FAP según la presencia de infección secundaria, la forma clínica del dengue y los niveles de factor de necrosis tumoral alfa (FNT-a), interferón gamma (IFN- $\gamma$ ) e interleucina 6 (IL-6).

\section{Materiales y métodos}

\section{Diseño del estudio}

Se llevó a cabo un estudio descriptivo y longitudinal, desde mayo de 2005 hasta marzo de 2007, en los departamentos de Antioquia y Chocó, Colombia.

\section{Población de estudio}

La población de estudio estuvo conformada por 43 pacientes mestizos y 33 afrodescendientes que cumplían con la definición de caso de la Organización Panamericana de la Salud/Organización Mundial de la Salud (44) y se confirmaron por laboratorio utilizando anticuerpos IgM en pruebas pareadas.

Los pacientes mestizos procedían de instituciones de salud del área metropolitana de Medellín y los pacientes afrodescendientes procedían de la región del Urabá antioqueño y del municipio de Quibdó. La clasificación étnica se basó en las características del cabello, las de la cara y el color de piel.

\section{Diagnóstico de laboratorio}

La infección por el virus del dengue se confirmó mediante la detección de anticuerpos IgM contra el virus en una muestra de la fase aguda y otra de la fase de convalecencia, utilizando un estuche comercial Dengue IgM Capture ELISA® (Panbio, Sinnamon Park, Australia). La infección secundaria se determinó por la detección de anticuerpos lgG contra el virus del dengue en la muestra de la fase aguda de la enfermedad, usando una prueba comercial UMELISA® Dengue IgG (IPK, Cuba).

El suero de los pacientes fue rápidamente separado y refrigerado a $-20{ }^{\circ} \mathrm{C}$ y almacenado a $-70{ }^{\circ} \mathrm{C}$ en el Instituto Colombiano de Medicina Tropical-Universidad CES, hasta su uso. La actividad de la AH-FAP se determinó en muestras de suero tomadas diariamente por cinco días en la fase aguda de la enfermedad ( 1 a 13 días del inicio de la enfermedad) y otra muestra tomada en la de convalecencia (14 a 21 días del inicio de la enfermedad), por medio de espectrofotometría y utilizando un estuche comercial Azwell Auto PAF$\mathrm{AH}$ kit $\AA$ (Alfresa Pharma Corporation, Osaka, Japan), siguiendo el protocolo del fabricante. En estos mismos pacientes se había hecho, en un estudio previo, la medición de los niveles en suero de las citocinas FNT-a, IFN- $\gamma$ e IL-6 (44), los cuales se compararon con la actividad de la AH-FAP. 


\section{Análisis estadístico}

El análisis estadístico se hizo con el programa Statistical Package for Social Sciences, SPSS $\AA_{\text {, }}$ versión 15, (Chicago, IL). Los datos cualitativos se expresaron en frecuencias absolutas y relativas $y$, los cuantitativos, en mediana y en percentil 25 (Q1) y percentil 75 (Q3). La diferencia de proporciones entre grupos se analizó mediante la prueba de $\mathrm{ji}^{2}$. La comparación estadística de la actividad sérica de la AH-FAP, entre los grupos étnicos y las formas clínicas, se hizo mediante la prueba no paramétrica de Mann-Whitney y, entre la fase aguda y la de convalecencia, se hizo mediante la prueba de Wilcoxon. La correlación entre el recuento de plaquetas, los niveles de citocinas y la actividad sérica de la AH-FAP, se estimó mediante el coeficiente de correlación de Spearman. El nivel de significancia se fijó en menor de 0,05.

\section{Aspectos éticos}

El proyecto fue aprobado por el Comité de Bioética del Instituto Colombiano de Medicina Tropical, Universidad CES. La participación en el estudio fue voluntaria y a cada participante se le solicitó consentimiento informado y escrito. Los procedimientos estuvieron de acuerdo con lo establecido en la Resolución número 8430 de 1993 del Ministerio de Salud de Colombia.

\section{Resultados}

\section{Aspectos generales}

La actividad de la AH-FAP se midió en 76 pacientes: 43 mestizos y 33 afrodescendientes. Los dos grupos étnicos no tuvieron diferencias según el sexo, pero sí según la edad. La proporción de hombres mestizos y afrodescendientes fue de $48,8 \%$ y de $48,5 \%$, respectivamente $(p=0,975)$. La mediana de la edad en los pacientes mestizos fue 23 años $y$, en los afrodescendientes, de 18 años $(p=0,001)$.

Hubo 14 (18\%) casos de dengue hemorrágico y fue más frecuente en los mestizos (23,3\% Vs. $12,1 \%)$, sin diferencias estadísticamente significativas. Los signos y síntomas clínicos generales más frecuentes, fueron: fiebre, cefalea y mialgias/artralgias (100\%, 97,4 \% y $86,8 \%$, respectivamente), y las manifestaciones hemorrágicas más frecuentes fueron: petequias (50 \%) y hemorragia gingival $(19,7$ $\%)$. Más mestizos que afrodescendientes requirieron hospitalización: 88,4 \% Vs. 59,8 \% ( $p=0,006)$.

El recuento de plaquetas [Q2 (Q1-Q3)] fue estadísticamente menor en mestizos que en afrodescendientes: 59.500 por $\mathrm{mm}^{3}$ (33.750-102.250)
Vs. 94.000 por $\mathrm{mm}^{3}$ (45.500-138.500) $(\mathrm{p}=0,005)$; y la mediana del hematocrito fue estadísticamente más alta en los pacientes mestizos: $43,2 \%(40,0-46,4)$ Vs. 38,1\% $(35,2-41,5)(p=0,000)$.

En general, hubo infección secundaria en $71,1 \%$ (54/76) de los casos. Al comparar esta variable entre los grupos étnicos, no se observaron diferencias estadísticamente significativas: 62,8 \% (27/43) en mestizos Vs. 81,8\% (27/33) en afrodescendientes $(p=0,07)$.

\section{Actividad sérica de la AH-FAP según grupo étnico y forma clínica}

La actividad en suero de la AH-FAP fue mayor en la fase aguda que en la de convalecencia $(0,788 \pm 0,485$ Vs. $0,768 \pm 0,428)$, sin diferencias estadísticamente significativas, $(p=0,194)$. Fue más elevada en afrodescendientes que en mestizos $(p=0,000)$, situación que se repite en ambas formas clínicas, con diferencias estadísticamente significativas en el dengue clásico.

La actividad de la AH-FAP en los mestizos fue más elevada en los casos de dengue hemorrágico que en los de dengue clásico $(p=0,012)$. En los afrodescendientes sucedió lo contrario, aquellos con dengue clásico presentaron mayor actividad de la AH-FAP, sin diferencias significativas con los de dengue hemorrágico. Para el total de casos, no hubo diferencias estadísticamente significativas de la actividad de esta enzima entre las formas clínicas (cuadro 1).

\section{Cinética de la actividad de la AH-FAP según grupos étnicos y forma clínica}

La actividad de la AH-FAP en los pacientes afrodescendientes fue elevada durante todo el período de observación, con un leve descenso el día diez. Por el contrario, en los mestizos la actividad durante los primeros días fue baja, para luego incrementarse en forma paulatina (figura 1a). La cinética de la AH-FAP, de acuerdo con la forma clínica y para el total de los pacientes, fue similar durante el período de observación (figura 1b). En los casos de dengue clásico y dengue hemorrágico, la actividad de la enzima fue elevada en afrodescendientes hasta el día ocho, tendencia más evidente en los casos de dengue clásico (figuras 1c y1d). Con relación al período crítico, del día 4 al 7 desde el inicio de los síntomas, se observó mayor actividad de la AH-FAP en los afrodescendientes que en los mestizos, tanto para su número total (figura 1a) como para las dos formas de la enfermedad (figuras 1c y1d). 
Cuadro 1. Actividad en suero $(\mathrm{U} / \mathrm{L})$ de la acetilhidrolasa del factor activador de plaquetas (AH-FAP) en pacientes con dengue, según grupo étnico y forma clínica

\begin{tabular}{|c|c|c|c|c|}
\hline \multirow[t]{2}{*}{ Forma clínica } & Mestizos & \multirow{2}{*}{$\begin{array}{c}\text { Afrodescendientes } \\
\text { (n) Mediana } \\
\text { (Q1-Q3) }\end{array}$} & \multirow{2}{*}{$\begin{array}{c}\text { Total } \\
\text { (n) Mediana } \\
\text { (Q1-Q3) }\end{array}$} & \multirow{2}{*}{$\begin{array}{c}\text { Mann-Whitney } \\
\text { P }\end{array}$} \\
\hline & $\begin{array}{l}\text { (n) Mediana } \\
\text { (Q1-Q3) }\end{array}$ & & & \\
\hline Dengue clásico & $\begin{array}{l}(33) 0,73 \\
(0,0-1,05)\end{array}$ & $\begin{array}{c}(29) 0,89 \\
(0,73-1,10)\end{array}$ & $\begin{array}{c}(62) 0,84 \\
(0,57-1,07)\end{array}$ & 0,000 \\
\hline Dengue hemorrágico & $\begin{array}{c}(10) 0,83 \\
(0,71-1,08)\end{array}$ & $\begin{array}{c}(4) 0,88 \\
(0,69-1,12)\end{array}$ & $\begin{array}{c}(14) 0,87 \\
(0,71-1,12\end{array}$ & 0,893 \\
\hline Total casos & $\begin{array}{l}(43) 0,76 \\
(0,0-1,03)\end{array}$ & $\begin{array}{c}(33) 0,89 \\
(0,72-1,10)\end{array}$ & $\begin{array}{c}(76) 0,84 \\
(0,57-1,07)\end{array}$ & 0,000 \\
\hline $\begin{array}{l}\text { Mann-Whitney } \\
\text { P }\end{array}$ & 0.012 & 0.600 & 0,201 & \\
\hline Dengue clásico Vs. de & hemorrágico & & & \\
\hline
\end{tabular}

$\mathrm{N}$ : número de casos; Q1: percentil 25; Q3:percentil 75.

Correlación entre la actividad sérica de la $\mathrm{AH}$ FAP y el conteo de plaquetas

Se observó una muy pequeña correlación positiva entre el conteo de plaquetas y la actividad sérica de la AH-FAP en todos los pacientes $(r=0,199)$. En ambos grupos étnicos la correlación fue positiva, muy baja en los mestizos $(r=0,174)$ y medio alta $(r=0,62)$ en los afrodescendientes; la variación en el recuento de plaquetas puede explicar la variación en los niveles de la AH-FAP, en $38 \%$ de los afrodescendientes.

\section{Actividad en suero de la AH-FAP e infección secundaria}

No se observaron diferencias estadísticamente significativas en la actividad de la AH-FAP, entre infección primaria y secundaria, para el total de los pacientes: $0,918(0,139-1,119)$ Vs. 0,879 (0,5961,048).

Correlación entre la actividad de la AH-FAP y los niveles séricos de citocinas

En todos los pacientes, se observó una correlación positiva entre la actividad sérica de la AH-FAP y los niveles de FNT-a $(r=0,126)$ y, una negativa, entre la actividad en suero de la AH-FAP y los niveles de IFN- $\gamma$ y de IL-6, $(r=-, 149)$ para IFN- $\gamma$ y $(r=-0,140)$ para IL-6, respectivamente. Ninguna de estas citocinas pudo explicar en más de $3 \%$ la variación de la actividad de la enzima. En ningún grupo étnico se encontró correlación entre la $\mathrm{AH}$ FAP y dichas citocinas.

\section{Discusión}

El presente estudio permitió observar diferencias en la respuesta clínica e inmunitaria entre dos grupos étnicos con infección por el virus del dengue. Los pacientes mestizos requirieron ser hospitalizados con mayor frecuencia, su conteo de plaquetas fue menor y su hematocrito fue mayor; de igual modo, los casos de dengue hemorrágico fueron más frecuentes en este grupo (10 Vs. 4). Estos datos concuerdan con otros estudios en los que los afrodescendientes presentan formas menos graves de dengue (11,21-24).

Se encontró mayor actividad sérica de la AH-FAP durante la fase aguda que en la de convalecencia, lo cual está a favor de que esta enzima se produzca durante la infección por el virus del dengue. Este hallazgo coincide con lo reportado por otros autores (43).

La actividad sérica de la AH-FAP fue significativamente mayor en los pacientes afrodescendientes que en los mestizos, diferencia que se mantiene para ambas formas clínicas, con diferencias estadísticamente significativas para el dengue clásico (cuadro 1). En los casos de dengue hemorrágico, las diferencias no fueron significativas debido, posiblemente, al limitado número de casos en afrodescendientes.

Según lo descrito en la literatura científica, la rápida degradación del factor activador de plaquetas por la enzima AH-FAP, es uno de los mecanismos de regulación de sus niveles (27), por lo cual proponen que la disminución plasmática de la enzima causa acumulación del factor activador de plaquetas, lo que conduce a estados patológicos (45). Por el contrario, otras enfermedades se han relacionado con elevación de la actividad de la AH-FAP, casos en los cuales se considera como una respuesta fisiológica a estímulos inflamatorios, 
uno de los mecanismos de defensa contra estas enfermedades (27).

Teniendo en cuenta las consideraciones anteriores a los hallazgos del presente estudio, se puede sugerir que la mayor actividad de la enzima en los pacientes afrodescendientes en comparación con los mestizos, tiene un efecto protector frente al desarrollo de formas graves del dengue, lo cual se manifiesta en un menor número de casos de dengue hemorrágico y menor frecuencia de manifestaciones graves del dengue en el grupo de los afrodescendientes. Igualmente, en este grupo la actividad sérica de la AH-FAP fue mayor en los casos de dengue clásico que en los de dengue hemorrágico.

Se observó lo contrario en los pacientes mestizos, en quienes la actividad sérica de la enzima fue significativamente mayor en el dengue hemorrágico que en el dengue clásico, lo cual se podría interpretar como una respuesta fisiológica a la infección, como se describió antes. Igualmente, esta observación apoya la presencia de una respuesta inmunológica diferente según la raza. Esta observación parece estar sustentada por la cinética de la enzima (figura 1a) descrita en este estudio, la cual es muy diferente entre grupos étnicos. En los mestizos, la actividad de la enzima es baja al inicio de la infección y aumenta al final, mientras que, en los afrodescendientes, es más elevada y constante en todo el período de la enfermedad.

Está demostrado que las citocinas proinflamatorias FNT-a, IL-6 e IFN- $\gamma$ son producidas durante la infección por el virus del dengue y se relacionan con la gravedad de la enfermedad (14-18), pero poco se conoce de su modulación por otros mediadores, como el factor activador de plaquetas, en la infección por dengue. En otras enfermedades, como en el accidente cerebrovascular, la expresión de FNT-a puede estimular la liberación del factor activador de plaquetas (46). Por otro lado, el tratamiento de
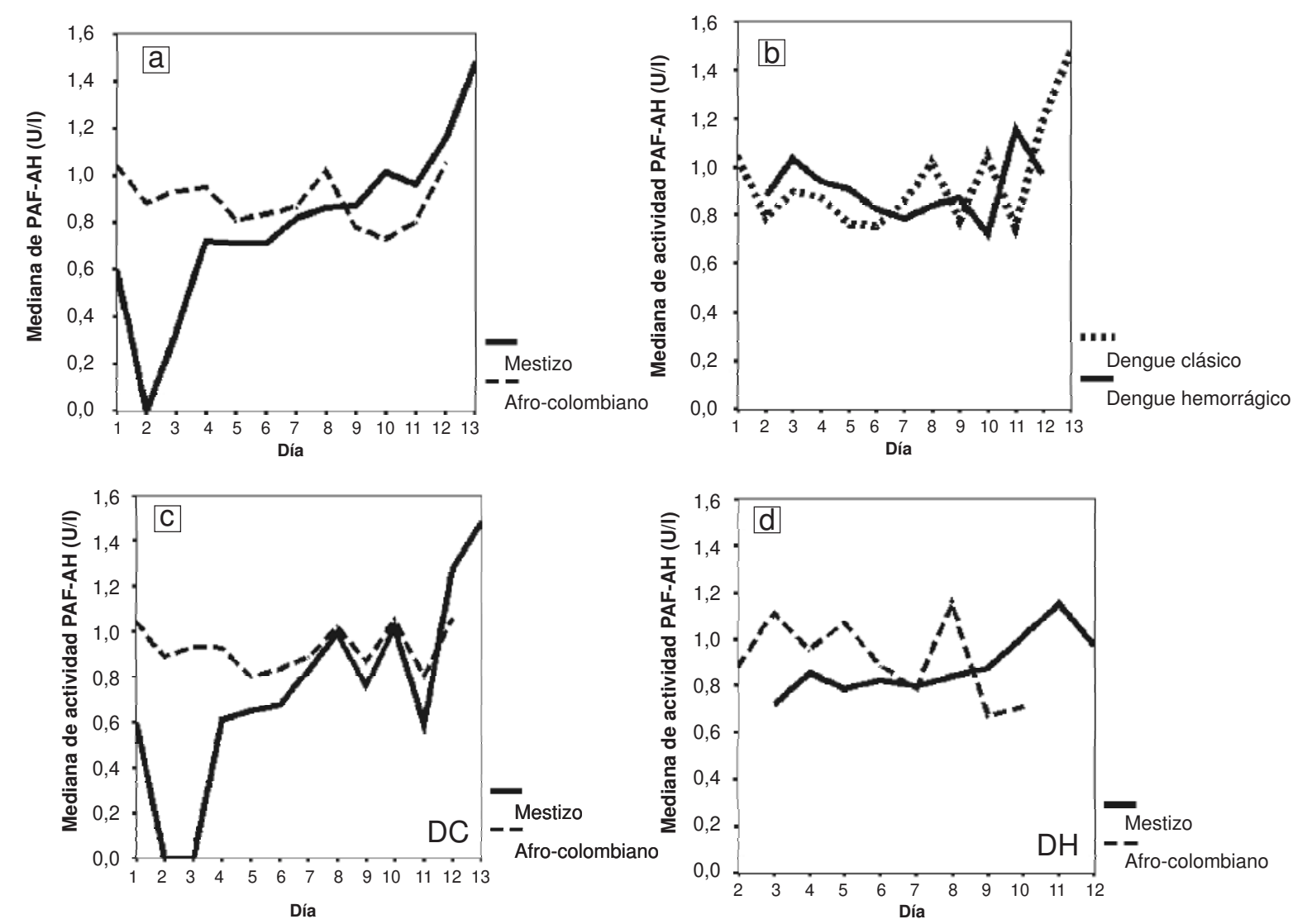

Figura 1. Cinética de la actividad sérica en U/L de la acetilhidrolasa del factor activador de plaquetas (AH-FAP) en pacientes con dengue, según grupo étnico y forma clínica

a. Actividad de la AH-FAP comparada entre grupos étnicos en el total de los casos. b. Actividad de la AH-FAP comparada entre formas clínicas de dengue en el total de los casos. c. Actividad de la AH-FAP comparada entre grupos étnicos en los casos de dengue clásico (DC). d. Actividad de la AH-FAP comparada entre grupos étnicos en los casos de dengue hemorrágico (DH). 
ratones con enfermedad inflamatoria con AH-FAP recombinante produce disminución de los niveles de IL-6 (34).

En el presente estudio, se encontró una correlación positiva entre la actividad sérica de AH-FAP y los niveles de FNT-a y una correlación negativa con los niveles de IL-6 e IFN- $\gamma$ Sin embargo, con los resultados obtenidos en el presente estudio y con los disponibles en la literatura científica, aún no es posible presentar conclusiones acerca de la interrelación de estas moléculas en los casos de infección por dengue.

En el presente estudio se encontraron diferencias en la actividad de la AH-FAP entre dos grupos étnicos con infección por el virus del dengue, lo cual concuerda con los hallazgos descritos sobre otros mediadores inmunológicos y sobre la respuesta a otras enfermedades virales. En pacientes con infección por el virus de la inmunodeficiencia humana (VIH) (47) y hepatitis C (48), se detectaron niveles más elevados de FNT-a en afroamericanos que en caucásicos. También, se han observado diferencias en la eliminación del virus de la hepatitis C relacionadas con la raza (49), así como diferencias en el curso de la enfermedad y la respuesta al tratamiento para hepatitis $C$ en afrodescendientes (50). En estos mismos pacientes, en un estudio previo, se midieron los niveles en suero de FNT-a, interferón IFN- $\gamma$ e IL-6. Los afrodescendientes presentaron niveles más elevados de FNT-a que los mestizos (51). También, está demostrado que la ascendencia africana es protectora contra las formas graves de dengue (52).

Una de las acciones del factor activador de plaquetas es el aumento de la agregación plaquetaria (41); por lo tanto, se espera que el conteo de plaquetas aumente al ser degradado por la AH-FAP. Esto podría constatarse con la correlación directa entre la actividad de la enzima y el conteo de plaquetas que se observó en los pacientes del presente estudio. Sin embargo, en otras enfermedades, como la inflamatoria intestinal, se describe lo contrario (28).

Los monocitos mononucleares infectados por DENV-1 (infección primaria), al ser expuestos a la infección por DENV-2 (infección secundaria), producen niveles más elevados de factor activador de plaquetas que los monocitos sin infección primaria expuestos a DENV-2 (41). Por lo tanto, es de esperarse diferencias en la actividad de la $\mathrm{AH}$ FAP entre estas dos infecciones. Sin embargo, en el presente estudio no se encontraron diferencias en la actividad de la enzima entre la infección primaria y la secundaria, lo cual sugiere la necesidad de explorar esta variable en un estudio con mayor número de casos.

Se ha documentado la relación de la mutación V279F con la deficiencia de la AH-FAP. En Japón, esta mutación prevalece en más de 30 $\%$ de la población encuestada y la presencia del alelo mutante es significativamente mayor en los pacientes con asma, accidente cerebrovascular, infarto de miocardio, hemorragia cerebral o miocardiopatía no familiar (53). Igualmente, el polimorfismo G994T, causante de la deficiencia de esta enzima, se ha asociado con el síndrome de ovario poliquístico en mujeres chinas (54).

En conclusión, los datos del presente estudio muestran diferencias en la respuesta inmunitaria a la infección por el virus del dengue entre grupos étnicos, con mayor producción de AH-FAP en los pacientes afrodescendientes, lo que apoya un papel protector de esta enzima en el desarrollo de formas graves del dengue. Sin embargo, la interpretación de esta asociación es difícil por el número limitado de casos y porque, como lo afirman algunos autores, la actividad de la AH-FAP puede verse afectada por los niveles de oxidación (55) y de lipoproteínas de baja densidad en el plasma (26). Por lo tanto, es necesario plantear nuevos proyectos para determinar la frecuencia de los polimorfismos relacionados con la producción de AH-FAP en estos grupos étnicos.

\section{Agradecimientos}

A Gladys Cartagena y a Darío Ibargüen, por su ayuda durante el trabajo de campo. A Amanda Maestre, por la revisión del manuscrito y a Cesar Segura, por el soporte científico.

\section{Conflicto de intereses}

Los autores no tienen ningún conflicto de intereses.

\section{Financiación}

COLCIENCIAS, Colombia. Proyecto No. 32560416286.

\section{Referencias}

1. Programa Especial para Investigación y Capacitación en Enfermedades Tropicales (TDR), Organización Mundial de la Salud. Dengue. Guías para el diagnóstico, tratamiento, prevención y control; 2009. p. 3. Fecha de consulta: 24 de enero de 2010. Disponible en: http:// whqlibdoc.who.int/publications/2009/9789995479213_ spa.pdf. 
2. Instituto Nacional de Salud. Estadística en Salud Pública. Vigilancia rutinaria. Fecha de consulta: 6 de abril 2011. Disponible en: http://www.ins.gov.co/ ?idcategoria $=85465 \#$.

3. Instituto Nacional de Salud. Boletín No. 29. Vigilancia epidemia por dengue en Colombia. Fecha de consulta: 23 de agosto 2010. Disponible en: http://new.paho.org/col/ index.php?option=com_content\&task=view\&id=810\&ltemid $=468$.

4. Kurane I. Dengue hemorrhagic fever with special emphasis on immunopathogenesis. Comp Immunol Microbiol Infect Dis. 2007;30:329-40.

5. Cardier JE, Mariño E, Romano E, Taylor P, Liprandi F, Bosch $\mathbf{N}$, et al. Proinflammatory factors present in sera from patients with acute dengue infection induce activation and apoptosis of human microvascular endothelial cells: Possible roll of TNF-a in endothelial cell damage in dengue. Cytokine. 2005;30:359-65.

6. Rosen L. The Emperor's new clothes revisited or reflections on the pathogenesis of dengue hemorrhagic fever. Am J Trop Med Hyg. 1997;26:337-43.

7. Ricco-Hesse R, Harrison LM, Salas RA, Tovar D, Nisalak A, Ramos C, et al. Origins of dengue type 2 viruses associated with increased pathogenicity in the Americas. Virology. 1997;230:244-51.

8. Balmaseda A, Hammond SN, Pérez L, Tellez Y, Saborío SI, Mercado JC, et al. Serotype-specific differences in clinical manifestations of dengue. Am J Trop Med Hyg. 2006;74:449-56.

9. Kumaria R. Correlation of disease spectrum among four dengue serotypes: A five years hospital based study from India. Braz J Infect Dis. 2010;14:141-6.

10. Murgue B, Roche C, Chungue E, Deparis X. Prospective study of the duration and magnitude of viraemia in children hospitalized during the 1996-1997 dengue-2 outbreak in French Polynesia. J Med Virol. 2000;60:432-8.

11. Guzmán MG, Kouri G, Valdés L, Bravo J, Álvarez M, Vásquez $\mathbf{S}$, et al. Epidemiological studies on dengue in Santiago de Cuba, 1997. Am J Epidemiol. 2000;152:793-9.

12. Halstead SB. Pathogenesis of dengue. Challenges to molecular biology. Science. 1988;238:476-81.

13. Rothman AL. Cellular immunology of sequential dengue virus infection and its role in disease pathogenesis. Curr Top Microbiol Immunol. 2010;338:83-98.

14. Chaturvedi UC, Elbishbishi EA, Agarwal R, Raghupathy $\mathbf{R}$, Nagar R, Tandon R, et al. Sequential production of cytokines by dengue virus-infected human peripheral blood leukocyte cultures. J Med Virol. 1999;59:335-40.

15. Juffrie M, Meer GM, Hack CE, Haasnoot K, Sutaryo, Veerman AJ. Inflammatory mediators in dengue virus infection in children interleukin-6 and its relation to C-reactive protein and secret phospholipase A2. Am J Trop Med Hyg. $2001 ; 65: 70-5$.

16. Pinto LM, Oliveira SO, Braga EL, Nogueira RM, Kubelka CF. Increased pro-inflamatory cytokines (TNF-alpha and IL-6) and anti-inflamatory compounds (sTNFRp55 and sTNFRp75) in Brazilian patients during exanthematic dengue fever. Mem Inst Oswaldo Cruz. 1999;94:387-94.
17. Hober D, Poli L, Roblin B, Gestas P, Chunge E, Granic G, et al. Serum levels of tumour necrosis factor-alpha (TNF-alpha), interleukin-6 (IL-6), and interleukin-1beta (IL1beta) in dengue-infected patients. Am J Trop Med Hyg. 1993;48:324-31.

18. Restrepo BN, Isaza DM, Salazar CL, Ramírez R, Ospina M, Álvarez LG. Serum levels of interleukin-6, tumor necrosis factor-alpha and interferon-gamma in infants with and without dengue. Rev Soc Bras Med Trop. 2008;41:610.

19. Halstead SB, Streit TG, Lafontant JG, Putvatana R, Russell K, Sun W, et al. Haiti: Absence of dengue hemorrhagic fever despite hyperendemic dengue virus transmission. Am J Trop Med Hyg. 2001;65:180-3.

20. Gubler DJ. Dengue and dengue hemorrhagic fever. Clin Microbiol Rev. 1998;11:480-96.

21. Rioth M, Beauharnais CA, Noel F, Ikizler MR, Mehta S, Zhu Y, et al. Serologic imprint of dengue virus in urban Haiti: Characterization of humoral immunity to dengue in infants and young children. Am J Trop Med Hyg. 2011;84:630-6.

22. Bravo J, Guzmán MG, Kouri G. Why dengue hemorrhagic in Cuba? 1. Individual risk factors for dengue hemorrhagic fever/dengue shock syndrome. Trans R Soc Trop Med Hyg. 1987;8:816-20.

23. Guzmán MG, Kouri GP, Vásquez S, Rosario D, Bravo JR, Valdés L. DHF epidemics in Cuba, 1981 and 1987: Some interesting observations. Dengue Bull WHO. 1999;23:39-43.

24. Sierra B, Kourí G, Guzmán MG. Race: A risk factor for dengue hemorrhagic fever. Arch Virol. 2007;152:533-42.

25. Shekhar KC, Huat OL. Epidemiology of dengue/dengue hemorrhagicfeverinMalaysia:Aretrospectiveepidemiological study 1973-1987. Part I: dengue hemorrhagic fever (DHF). Asia Pac J Public Health. 1992;6:15-25.

26. Kosaka T, Yamaguchi M, Soda Y, Kishimoto T, Tago A, Toyosato $\mathbf{M}$, et al. Spectrophotometric assay for serum platelet-activating factor acetylhydrolase activity. Clin Chim Acta. 2000;296:151-61.

27. Karasawa K, Harada A, Satoh N, Inoue K, Setaka M. Plasma platelet activating factor-acetylhydrolase (PAF-AH). Prog Lipid Res. 2003;42:93-114.

28. Oshimoto H, Okamura S, lida T, Ishikawa T, Hosaka $\mathrm{K}$, Mori M. Diagnostic value of the serum platelet-activating factor acetylhydrolase activity in inflammatory bowel disease. Tohoku J Exp Med. 2005;207:65-71.

29. Kosaka T, Yamaguchi M, Miyanaga K, Mizuno K. Serum platelet-activating factor acetyhydrolasa (PAF-AH) activity in more than 3000 healthy Japanese. Clin Chim Acta. 2001;312:179-83.

30. Karabina SA, Ninio E. Plasma PAF-acetylhydrolase: an unfulfilled promise? Biochim Biophys Acta. 2006;1761:1351-8.

31. Arai H, Koizumi H, Aoki J, Inoue K. Platelet-activating factor acetylhydrolase (PAF-AH). J Biochem. 2002;131:63540.

32. Lu J, Pierce M, Franklin A, Jilling T, Stafforini DM, Caplan M. Dual roles of endogenous platelet-activating factor acetylhydrolase in a murine model of necrotizing enterocolitis. Pediatr Res. 2010;68:225-30. 
33. Graham RM, Stephens CJ, Silvester W, Leong LL, Sturm MJ, Taylor RR. Plasma degradation of platelet-activating factor in severely ill patients with clinical sepsis. Crit Care Med. 1994;22:204-12.

34. Gomes RN, Bozza FA, Amâncio RT, Japiassú AM, Vianna RC, Larangeira AP, et al. Exogenous plateletactivating factor acetylhydrolase reduces mortality in mice with systemic inflammatory response syndrome and sepsis. Shock. 2006;26:41-9.

35. Miwa M, Miyake T, Yamanaka T, Sugatani J, Suzuki Y, Sakata S, et al. Characterization of serum platelet-activating factor (PAF) acetylhydrolase: Correlation between deficiency of serum PAF acetylhydrolase and respiratory symptoms in asthmatic children. J Clin Invest. 1988;82:1983-91.

36. Caini P, Guerra CT, Giannini C, Giannelli F, Gragnani L, Petrarca A, et al. Modifications of plasma platelet-activating factor (PAF)-acetylhydrolase/PAF system activity in patients with chronic hepatitis $C$ virus infection. J Viral Hepat. 2007;14:22-8.

37. Guerra CT, Caini P, Giannini C, Giannelli F, Gragnani L, Petrarca A, et al. Effect of chronic hepatitis $C$ virus infection on inflammatory lipid mediators. Dig Liver Dis. 2007;39 (Suppl.1):S76-82.

38. Satoh K, Imaizumi T, Kawamura Y, Yoshida H, Takamatsu S, Takamatsu M. Increased activity of platelet activating factor acetylhydrolase in plasma low density lipoprotein from patients with essential hypertension. Prostaglandins. 1989;37:673-82.

39. Satoh K, Yoshida H, Imaizumi T, Takamatsu S, Mizuno S. Platelet-activating factor acetylhydrolase in plasma lipoproteins from patients with ischemic stroke. Stroke. 1992;23:1091-2.

40. Hofmann B, Ruhling K, Spangenberg P, Ostermann G. Enhanced degradation of platelet-activating factor in serum from diabetic patients. Haemostasis. 1989;19:180-4.

41. Yang KD, Lee CS, Shaio MF. A higher production of platelet activating factor in ex vivo heterologously secondary dengue-2 virus infections. Acta Microbiol Immunol Hung. 1995;42:403-7.

42. Souza DG, Fagundes CT, Sousa LP, Amaral FA, Souza RS, Souza AL, et al. Essential role of platelet-activating factor receptor in the pathogenesis of dengue virus infection. Proc Natl Acad Sci USA. 2009;106:14138-43.

43. Seet RC, Lee CY, Lim EC, Quek AM, Yeo LL, Huang SH, et al. Oxidative damage in dengue fever. Free Radic Biol Med. 2009;47:375-80.
44. PAHO. Dengue hemorrhagic fever in the Americas: Guidelines for prevention and control. Scientific publication no. 548. Washington, D.C.: Panamerican Health Organization; 1995

45. Serebruany VL, Gurbel PA, Murugesan SR, Lowry DR, Sturm E, Svetlov SI. Depressed plasma platelet-activating factor acetylhydrolase in patients presenting with acute myocardial infarction. Cardiology.1998;90:127-30.

46. Tuttolomondo A, Di Raimondo D, Di Sciacca R, Pinto A, Licata G. Inflammatory cytokines in acute ischemic stroke. Curr Pharm Des. 2008;14:3574-89.

47. Sonnerborg A, Ayehunie S, Julander I. Elevated levels of circulating tumor necrosis alpha in human immunodeficiency virus type 1-infected Africans living in Sweden. Clin Diagn Lab Immunol. 1995;2:118-9.

48. Kimball T, Eslwick RK, Shiffman M. Ethnicity and cytokine production gauge responses of patients with hepatitis $C$ to interferon-alpha therapy. J Med Virol. 2001;65: 510-6.

49. Thio CL, Thomas DL, Goedert JJ, Vlahov D, Nelson KE, Hilgartner MW, et al. Racial differences in HLA class II associations with hepatitis $\mathrm{C}$ virus outcomes. J Infect Dis. 2001;184:16-21.

50. Jeffers LJ. Treating hepatitis $C$ in African Americans. Liver Int. 2007;27:313-22

51. Restrepo BN, Ramírez RE, Arboleda M, Álvarez G, Ospina M, Díaz FJ. Serum levels of cytokines in two ethnic groups with dengue virus infection. Am J Trop Med Hyg. 2008;79:673-7.

52. Blanton RE, Silva LK, Morato VG, Parrado AR, Dias JP, Melo PR, et al. Genetic ancestry and income are associated with dengue hemorrhagic fever in a highly admixed population. Eur J Hum Genet. 2008;16:762-65.

53. Tjoelker LW, Stafforini DM. Platelet-activating factor acetylhydrolases in health and disease. Biochim Biophys Acta. 2000;1488:102-23.

54. Fan P, Liu HW, Wang XS, Zhang F, Song Q, Li Q, et al. Identification of the G994T polymorphism in exon 9 of plasma platelet-activating factor acetylhydrolase gene as a risk factor for polycystic ovary syndrome. Hum Reprod. 2010;25:1288-94.

55. Ambrosio G, Oriente A, Napoli C, Palumbo G, Chiariello $\mathbf{P}$, Marone $\mathbf{G}$, et al. Oxygen radicals inhibit human plasma acetylhydrolase, the enzyme that catabolizes plateletactivating factor. J Clin Invest. 1994;93:2408-16. 